Theoretical Modelling on the Magnetization

by Electric Field Through Product Property

\title{
Z. Huang
}

Department of Materials, School of Applied Sciences, Cranfield University, Beds, MK43 0AL, UK

\begin{abstract}
Multilayer composites of piezoelectric and magnetostrictive materials can be designed to exhibit the magnetoelectric (ME) effect. This ME effect can be realised as an electric polarisation induced by a magnetic field (called $\mathrm{ME}_{\mathrm{H}}$ effect) or a magnetization by an electric field (called $\mathrm{ME}_{\mathrm{E}}$ effect). Theoretical modelling of the $\mathrm{ME}_{\mathrm{E}}$ effect for 2-2 connectivity composites has been developed for three different boundary conditions for perfect coupling at the interface. The calculated $\mathrm{ME}_{\mathrm{E}}$ coefficients using material properties of piezoelectric lead zirconate titanate (PZT) and magnetostrictive Terfenol-D are a few orders of magnitude larger than those of single phase ME materials and the calculated values are compared with experimental results in the literature.
\end{abstract}

Keywords: magnetoelectric, multiferroic, piezoelectric, magnetostrictive, piezomagnetic, laminated composites, modelling.

Author e-mail: Z.Huang@Cranfield.ac.uk 


\section{INTRODUCTION}

The magnetoelectric effect (ME) is characterized by the appearance of an electric polarization on applying a magnetic filed ( $\alpha=\delta \mathbf{P} / \delta \mathbf{H}$, called the $\mathrm{ME}_{\mathrm{H}}$ effect) or by the appearance of a magnetization on applying an electric field $\left(\alpha=\delta \mathbf{M} / \delta \mathbf{E}\right.$, called $\mathrm{ME}_{\mathrm{E}}$ effect). It was originally predicted by Landau and Lifshitz from thermodynamics and symmetry considerations ${ }^{1}$. This effect was first measured in a $\mathrm{Cr}_{2} \mathrm{O}_{3}$ crystal by a dynamic $\mathrm{ME}_{\mathrm{E}}$ method $^{2}$ and then confirmed with a $\mathrm{ME}_{\mathrm{H}}$ method ${ }^{3,4}$. The ME effect in single phase materials is usually very small and often can only be detected at very low temperatures, e.g., the ME coefficient $\alpha$ for $\mathrm{Cr}_{2} \mathrm{O}_{3}$ is no more than $4.13 \times 10^{-12}(\mathrm{~s} / \mathrm{m})$ at room temperature and the largest observed ME coefficient in single phase materials was seen in $\mathrm{TbPO}_{4}\left(36.7 \times 10^{-12}(\mathrm{~s} / \mathrm{m})\right)$ below $2.2 \mathrm{~K}^{5-6}$.

The ME effect can also be achieved through product property ${ }^{7-8}$, where the magneticelectric coupling in some magnetostrictive-piezoelectric composites could be a few orders larger ${ }^{7-17}$ than that in the single phase materials. Van Suchtelen proposed the product property concept, whereby entirely new properties could be achieved by coupling a pair of tensor properties in two materials that were intimately mixed ${ }^{7}$. Since then two main materials processing routes have been investigated to achieve ME effect this way, namely, (a) particulate magnetostrictive (MS) and piezoelectric (PE) composites and (b) laminated MS/PE composites. Investigations on eutectic coexisting phases of spinel cobalt ferrite-cobalt titanate and perovskite barium titanate achieved a magnetoelectric voltage coefficient $\alpha_{\mathrm{E}}\left(=\alpha / \varepsilon_{0} \varepsilon_{\mathrm{r}}\right)$ of $\sim 50 \mathrm{mV} / \mathrm{cm} \mathrm{Oe}$, despite the theoretical estimation being 40-60 times larger ${ }^{8}$, here $\varepsilon_{\mathrm{r}}$ is the relative 
permittivity and $\varepsilon_{0}$ the permittivity in vacuum. Similar ME coefficients were obtained with particulate composites of the giant MS rare-earth-iron alloy $\mathrm{Tb}_{1-}$ ${ }_{x} \mathrm{Dy}_{\mathrm{x}} \mathrm{Fe}_{2}$ (TFD) and lead zirconate titanate (PZT) and PVDF polymer ${ }^{9}$. A recent investigation of $\mathrm{CoFe}_{2} \mathrm{O}_{4}-\mathrm{BiFeO}_{3}$ nanocomposites obtained values for $\alpha_{\mathrm{E}}$ as high as $280 \mathrm{mV} / \mathrm{cm} \mathrm{Oe}^{10}$. On the other hand, laminated MS/PE structures have been found to show much higher $\mathrm{ME}_{\mathrm{H}}$ coefficients. Values for $\alpha_{\mathrm{E}}$ of $1500 \mathrm{mV} / \mathrm{cm}$ Oe for ferrite$\mathrm{PZT}^{11}$ and $4680 \mathrm{mV} / \mathrm{cm}$ Oe for TFD/PZT laminar structures ${ }^{12}$ have been reported. Significant enhancement of the $\mathrm{ME}_{\mathrm{H}}$ response can be obtained when the modulation frequency of the applied fields coincides with magnetic, electrical or mechanical eigenmodes of the system under study ${ }^{13-15}$. Partly because it is easier to realise, the published works on the ME effect as a product property in composite materials has so far concentrated entirely on the $\mathrm{ME}_{\mathrm{H}}$ effect, namely, a magnetic field $\mathrm{H}$ as the input and an electric voltage as the output.

Nowadays, electronic data is typically stored as regions of magnetic polarisation within ferromagnets and the magnetic polarisation can be reversed by magnetic fields. The manipulation of magnetisation by means other than magnetic fields for example electric fields could find applications in magnetic storage, spintronics ${ }^{18}$, and other magnetic sensing and actuating micro-electric-mechanical systems (MEMS) devices ${ }^{15}$. Recently electrically controlled magnetic switches in the hole-induced ferromagnetic semiconductor ( $\mathrm{In}, \mathrm{Mn}) \mathrm{As}^{19}$ and in the magnetic ferroelectric compound $\mathrm{HoMnO}_{3}{ }^{20}$ via magnetoelectric interaction, and the efficient coupling between ferroelectric and magnetic order parameters in the self-organised nanocomposite $\mathrm{BaTiO}_{3}-\mathrm{CoFe}_{2} \mathrm{O}_{4}{ }^{21}$ have been investigated. 
Recently, we have investigated the $\mathrm{ME}_{\mathrm{E}}$ effect in laminated MS/PE composites and observed a large induced magnetization by applying an electric field to the composites $^{22}$. This communication presents a theoretical calculation of the induced magnetisation by the electric field for the laminated MS/PE composites. For the low frequency response of the $\mathrm{ME}_{\mathrm{H}}$ effect, $\mathrm{Harshe}^{23}$ et. al developed theoretical models for MS/PE composites with 2-2 connectivity and obtained expressions for the longitudinal $\mathrm{ME}_{\mathrm{H}}$ coefficients by assuming an ideal coupling at the MS and PE interfaces. This model was improved to account for the non-perfect coupling at the interfaces by Bichurin, Petrov and Srinivasan ${ }^{24-26}$. There is no published work on the modelling of the $\mathrm{ME}_{\mathrm{E}}$ effect as a product property. In this paper, we will use the basic constituent equations for the PE and MS materials to obtain modelling results for the $\mathrm{ME}_{\mathrm{E}}$ coefficients for laminated MS/PE composites and compare these figures with experimental results in the literature.

\section{GENERAL THEORY}

We consider a bilayer consisting of magnetostrictive (MS) and piezoelectric (PE) materials. For many cases, both the MS and PE materials are polycrystalline, and therefore have the symmetry $\infty \mathrm{m}$. Usually, the electric polarisation or the easy magnetization direction (which is often the bias DC magnetic field direction as well) is assigned direction 3 in the Cartesian coordinate system so that the material property matrix can be defined. This will make it much easier for material scientists to assign values from the general property matrix to their material property parameters. For this purpose a Cartesian coordinate system is applied to the MS or PE phase and 3 is always the polarization or the easy magnetization direction. Table I summaries some 
symmetry relations for non-zero coefficients of piezoelectric and piezomagnetic phases. Only the linear ME effect is considered, any bilinear or higher order effects are ignored. We also ignore the hysteresis effect of magnetization ${ }^{27}$. Perfect coupling between the MS and PE layers is assumed.

We assume that there is no bending of the structure. In practice, this can be achieved by configuring a symmetric sandwich structure such as MS/PE/MS or PE/MS/PE, where the total cross sectional area of the MS (PE) layer accounts for the area of the MS (PE) phase.

For piezoelectric materials,

$$
\begin{aligned}
& { }^{p} S_{i}={ }^{p} S_{i j}^{E}{ }^{p} T_{j}+d_{k i} E_{k} \\
& D_{i}=d_{i j}^{p} T_{j}+\varepsilon_{i j} E_{j}
\end{aligned}
$$

Where ${ }^{\mathrm{p}} \mathrm{S}_{\mathrm{i}}$ and ${ }^{\mathrm{p}} \mathrm{T}_{\mathrm{j}}$ are the strain and stress tensor components of the PE phase, $\mathrm{E}_{\mathrm{k}}$ and $D_{i}$ are the vector components of electric field and electric displacement, ${ }^{p} s_{i j}^{E}$ are components of compliance at constant electric field of the PE phase, and $\mathrm{d}_{\mathrm{ij}}$ and $\varepsilon_{\mathrm{ij}}$ are components of piezoelectric coefficient and permittivity matrix respectively. Notice that the Einstein summation convention has been applied.

For piezomagnetive magnetostrictive materials, we have $\mathrm{e}^{28}$

$$
\begin{gathered}
{ }^{m} S_{i}={ }^{m} s_{i j}^{H}{ }^{m} T_{j}+q_{k i} H_{k} \\
B_{i}=q_{i j}{ }^{m} T_{j}+\mu_{i j} H_{j}
\end{gathered}
$$

Where ${ }^{\mathrm{m}} \mathrm{S}_{\mathrm{i}}$ and ${ }^{\mathrm{m}} \mathrm{T}_{\mathrm{j}}$ are the strain and stress tensor components of the MS phase, $\mathrm{H}_{\mathrm{k}}$ and $\mathrm{B}_{\mathrm{i}}$ are the vector components of magnetic field and magnetic induction, ${ }^{m} s_{i j}^{H}$ are 
components of compliance at constant magnetic field of the MS phase, and $\mathrm{q}_{\mathrm{ij}}$ and $\mu_{\mathrm{ij}}$ are components of piezomagnetic coefficient and permeability matrix respectively.

If a DC bias field of $\mathbf{H}_{\mathbf{0}}$, and a small AC field of $\boldsymbol{\delta E} \neq 0$ and $\boldsymbol{\delta H}=0$ are applied, then the above equations for $\mathrm{AC}$ response become

$$
\begin{aligned}
& \delta^{p} S_{i}={ }^{p} S_{i j}^{E} \delta^{p} T_{j}+d_{k i} \delta E_{k} \\
& \delta^{m} S_{i}={ }^{m} s_{i j}^{H} \delta^{m} T_{j} \\
& \delta B_{i}=q_{i j} \delta^{m} T_{j}
\end{aligned}
$$

These are the basic equations of this work. For simplicity, the terms $\delta \mathrm{S}_{\mathrm{ij}}, \delta \mathrm{T}_{\mathrm{ij}}, \delta \mathrm{E}_{\mathrm{i}}$, and $\delta B_{i}$ will be written as $S_{i j}, T_{i j}, E_{i}$ and $B_{i}$ in the following sections. From (3) the detailed equations are:

$$
\begin{aligned}
& { }^{p} S_{1}={ }^{p} S_{11}^{E}{ }^{p} T_{1}+{ }^{p} S_{12}^{E}{ }^{p} T_{2}+{ }^{p} S_{13}^{E}{ }^{p} T_{3}+d_{31} E_{3} \\
& { }^{p} S_{2}={ }^{p} S_{21}^{E} T_{1}+{ }^{p} S_{22}^{E}{ }^{p} T_{2}+{ }^{p} s_{23}^{E} T_{3}+d_{31} E_{3} \\
& { }^{m} S_{1}={ }^{m} S_{11}^{H} T_{1}+{ }^{m} S_{12}^{H}{ }^{m} T_{2}+{ }^{m} s_{13}^{H} T_{3} \\
& { }^{m} S_{2}={ }^{m} s_{21}^{H} T_{1}+{ }^{m} s_{22}^{H} T_{2}+{ }^{m} s_{23}^{H}{ }^{m} T_{3} \\
& B_{1}=q_{11}{ }^{m} T_{1}+q_{12}{ }^{m} T_{2}+q_{13}{ }^{m} T_{3} \\
& B_{2}=q_{21}{ }^{m} T_{1}+q_{22}{ }^{m} T_{2}+q_{23}{ }^{m} T_{3} \\
& B_{3}=q_{31}{ }^{m} T_{1}+q_{32}{ }^{m} T_{2}+q_{33}{ }^{m} T_{3}
\end{aligned}
$$

Three cases will now be discussed, which represent (i) the longitudinal, (ii) the transverse and (iii) the in-plane orientations, as shown schematically in Fig. 1.

\section{MAGNETOELECTRIC EFFECTS IN THE LAMINATED}

MAGNETOSTRICTIVE-PIEZOELECTRIC LAYERS

Case (i): Longitudinal orientation

The boundary condition for this case is ${ }^{\mathrm{m}} \mathrm{T}_{3}={ }^{\mathrm{p}} \mathrm{T}_{3}=0$. For balanced force conditions we have ${ }^{m} A_{1}{ }^{m} T_{1}+{ }^{p} A_{1}{ }^{p} T_{1}=0$ and ${ }^{m} A_{2}{ }^{m} T_{2}+{ }^{p} A_{2}{ }^{p} T_{2}=0$ where ${ }^{m} A_{1}\left({ }^{m} A_{2}\right)$ and ${ }^{p} A_{1}\left({ }^{p} A_{2}\right)$ 
are the section areas across the direction 1 (2) for the MS and PE layers respectively. Assuming perfect coupling at the interface, ${ }^{\mathrm{m}} \mathrm{S}_{1}={ }^{\mathrm{p}} \mathrm{S}_{1}$ and ${ }^{\mathrm{m}} \mathrm{S}_{2}={ }^{\mathrm{p}} \mathrm{S}_{2}$.

Solving equation (4) with the above boundary conditions leads to:

$$
\begin{aligned}
& \alpha_{13}=\frac{B_{1}}{E_{3}}=\frac{d_{31}\left[\left({ }^{m} s_{12}^{H}+{ }^{p} s_{12}^{E} \frac{{ }^{m} A_{2}}{{ }^{p} A_{2}}-{ }^{m} s_{11}^{H}-{ }^{p} s_{11}^{E} \frac{{ }^{m} A_{2}}{{ }^{p} A_{2}}\right) q_{11}+\left({ }^{m} s_{12}^{H}+{ }^{p} s_{12}^{E} \frac{{ }^{m} A_{1}}{{ }^{p} A_{1}}-{ }^{m} s_{11}^{H}-{ }^{p} s_{11}^{E} \frac{{ }^{m} A_{1}}{p^{p} A_{1}}\right) q_{12}\right]}{\left[\left({ }^{m} s_{12}^{H}+{ }^{p} s_{12}^{E} \frac{{ }^{m} A_{1}}{{ }^{p} A_{1}}\right)\left({ }^{m} s_{12}^{H}+{ }^{p} s_{12}^{E} \frac{{ }^{m} A_{2}}{{ }^{p} A_{2}}\right)-\left({ }^{m} s_{11}^{H}+{ }^{p} s_{11}^{E} \frac{{ }^{m} A_{1}}{{ }^{p} A_{1}}\right)\left({ }^{m} s_{11}^{H}+{ }^{p} s_{11}^{E} \frac{{ }^{m} A_{2}}{{ }^{p} A_{2}}\right)\right]} \\
& \alpha_{23}=\frac{B_{2}}{E_{3}}=\frac{d_{31}\left[\left({ }^{m} s_{12}^{H}+{ }^{p} s_{12}^{E} \frac{{ }^{m} A_{2}}{{ }^{p} A_{2}}-{ }^{m} s_{11}^{H}-{ }^{p} s_{11}^{E} \frac{{ }^{m} A_{2}}{{ }^{p} A_{2}}\right) q_{12}+\left({ }^{m} s_{12}^{H}+{ }^{p} s_{12}^{E} \frac{{ }^{m} A_{1}}{{ }^{p} A_{1}}-{ }^{m} s_{11}^{H}-{ }^{p} s_{11}^{E} \frac{{ }^{m} A_{1}}{{ }^{p} A_{1}}\right) q_{11}\right]}{\left[\left({ }^{m} s_{12}^{H}+{ }^{p} s_{12}^{E} \frac{{ }^{m} A_{1}}{{ }^{p} A_{1}}\right)\left({ }^{m} s_{12}^{H}+{ }^{p} s_{12}^{E} \frac{{ }^{m} A_{2}}{{ }^{p} A_{2}}\right)-\left({ }^{m} s_{11}^{H}+{ }^{p} s_{11}^{E} \frac{{ }^{m} A_{1}}{{ }^{p} A_{1}}\right)\left({ }^{m} s_{11}^{H}+{ }^{p} s_{11}^{E} \frac{{ }^{m} A_{2}}{{ }^{p} A_{2}}\right)\right]} \\
& \alpha_{33}=\frac{B_{3}}{E_{3}}=\frac{d_{31} q_{31}\left[\left({ }^{m} S_{12}^{H}+{ }^{p} S_{12}^{E} \frac{{ }^{m} A_{2}}{{ }^{p} A_{2}}-{ }^{m} S_{11}^{H}-{ }^{p} S_{11}^{E} \frac{{ }^{m} A_{2}}{{ }^{p} A_{2}}\right)+\left({ }^{m} S_{12}^{H}+{ }^{p} S_{12}^{E} \frac{{ }^{m} A_{1}}{{ }^{p} A_{1}}-{ }^{m} S_{11}^{H}-{ }^{p} S_{11}^{E} \frac{{ }^{m} A_{1}}{{ }^{p} A_{1}}\right)\right]}{\left[\left({ }^{m} S_{12}^{H}+{ }^{p} S_{12}^{E} \frac{{ }^{m} A_{1}}{{ }^{p} A_{1}}\right)\left({ }^{m} S_{12}^{H}+{ }^{p} S_{12}^{E} \frac{{ }^{m} A_{2}}{{ }^{p} A_{2}}\right)-\left({ }^{m} S_{11}^{H}+{ }^{p} S_{11}^{E} \frac{{ }^{m} A_{1}}{{ }^{p} A_{1}}\right)\left({ }^{m} S_{11}^{H}+{ }^{p} S_{11}^{E} \frac{{ }^{m} A_{2}}{{ }^{p} A_{2}}\right)\right]}
\end{aligned}
$$

Case (ii): Transverse orientation

For this case, the boundary conditions are: ${ }^{\mathrm{p}} \mathrm{T}_{3}={ }^{\mathrm{m}} \mathrm{T}_{2}=0,{ }^{\mathrm{m}} \mathrm{S}_{3}={ }^{\mathrm{p}} \mathrm{S}_{1},{ }^{\mathrm{m}} \mathrm{S}_{1}={ }^{\mathrm{p}} \mathrm{S}_{2}$ and the force balance conditions are ${ }^{m} A_{3}{ }^{m} T_{3}+{ }^{p} A_{1}{ }^{p} T_{1}=0$ and ${ }^{m} A_{1}{ }^{m} T_{1}+{ }^{p} A_{2}{ }^{p} T_{2}=0$. Similar to the case (i), the results are:

$$
\begin{aligned}
& \alpha_{13}=\frac{B_{1}}{E_{3}}=\frac{d_{31}\left[\left({ }^{m} S_{33}^{H}+{ }^{p} S_{11}^{E} \frac{{ }^{m} A_{3}}{{ }^{p} A_{1}}-{ }^{m} S_{31}^{H}-{ }^{p} S_{12}^{E} \frac{{ }^{m} A_{3}}{{ }^{p} A_{1}}\right) q_{11}+\left({ }^{m} S_{11}^{H}+{ }^{p} S_{11}^{E} \frac{{ }^{m} A_{1}}{{ }^{p} A_{2}}-{ }^{m} S_{31}^{H}-{ }^{p} S_{12}^{E} \frac{{ }^{m} A_{1}}{{ }^{p} A_{2}}\right) q_{13}\right]}{\left[\left({ }^{m} S_{11}^{H}+{ }^{p} S_{11}^{E} \frac{{ }^{m} A_{1}}{p^{p} A_{2}}\right)\left({ }^{m} S_{33}^{H}+{ }^{p} S_{11}^{E} \frac{{ }^{m} A_{3}}{{ }^{p} A_{1}}\right)-\left({ }^{m} S_{31}^{H}+{ }^{p} S_{12}^{E} \frac{{ }^{m} A_{1}}{{ }^{p} A_{2}}\right)\left({ }^{m} S_{31}^{H}+{ }^{p} S_{12}^{E} \frac{{ }^{m} A_{3}}{{ }^{p} A_{1}}\right)\right]}
\end{aligned}
$$

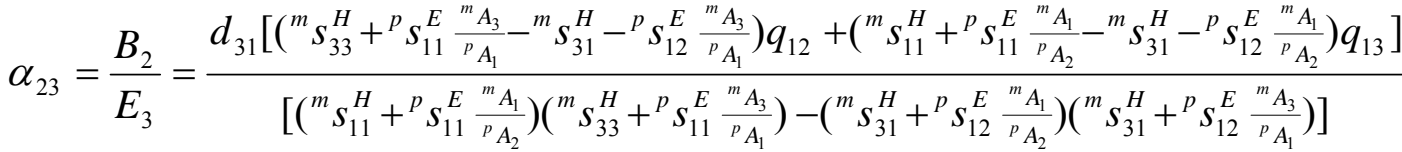

$$
\begin{aligned}
& \alpha_{33}=\frac{B_{3}}{E_{3}}=\frac{d_{31}\left[\left({ }^{m} S_{33}^{H}+{ }^{p} S_{11}^{E} \frac{{ }^{m} A_{3}}{{ }^{p} A_{1}}-{ }^{m} S_{31}^{H}-{ }^{p} S_{12}^{E} \frac{{ }^{m} A_{3}}{{ }^{p} A_{1}}\right) q_{31}+\left({ }^{m} S_{11}^{H}+{ }^{p} S_{11}^{E} \frac{{ }^{m} A_{1}}{{ }^{p} A_{2}}-{ }^{m} S_{31}^{H}-{ }^{p} S_{12}^{E} \frac{{ }^{m} A_{1}}{{ }^{p} A_{2}}\right) q_{33}\right]}{\left[\left({ }^{m} S_{11}^{H}+{ }^{p} S_{11}^{E} \frac{{ }^{m} A_{1}}{p^{p} A_{2}}\right)\left({ }^{m} S_{33}^{H}+{ }^{p} S_{11}^{E} \frac{{ }^{m} A_{3}}{{ }^{p} A_{1}}\right)-\left({ }^{m} S_{31}^{H}+{ }^{p} S_{12}^{E} \frac{{ }^{m} A_{1}}{{ }^{p} A_{2}}\right)\left({ }^{m} S_{31}^{H}+{ }^{p} S_{12}^{E} \frac{{ }^{m} A_{3}}{{ }^{p} A_{1}}\right)\right]}
\end{aligned}
$$


Case (iii): In-plane orientation

For this case, the boundary conditions are: ${ }^{\mathrm{p}} \mathrm{T}_{2}={ }^{\mathrm{m}} \mathrm{T}_{2}=0,{ }^{\mathrm{m}} \mathrm{S}_{3}={ }^{\mathrm{p}} \mathrm{S}_{3},{ }^{\mathrm{m}} \mathrm{S}_{1}={ }^{\mathrm{p}} \mathrm{S}_{1}$ and the force balance conditions are ${ }^{m} A_{3}{ }^{m} T_{3}+{ }^{p} A_{3}{ }^{p} T_{3}=0$ and ${ }^{m} A_{1}{ }^{m} T_{1}+{ }^{p} A_{1}{ }^{p} T_{1}=0$. Similar to the case (i), the results are:

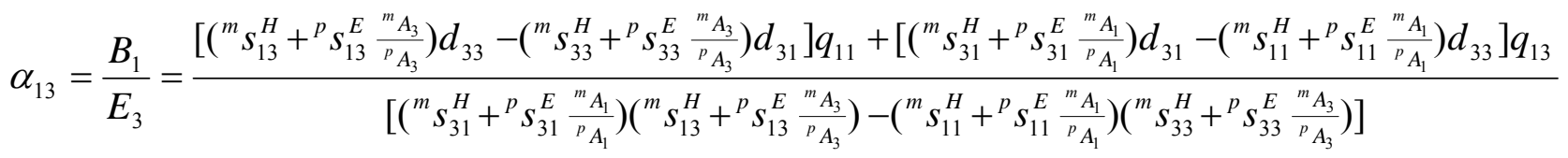

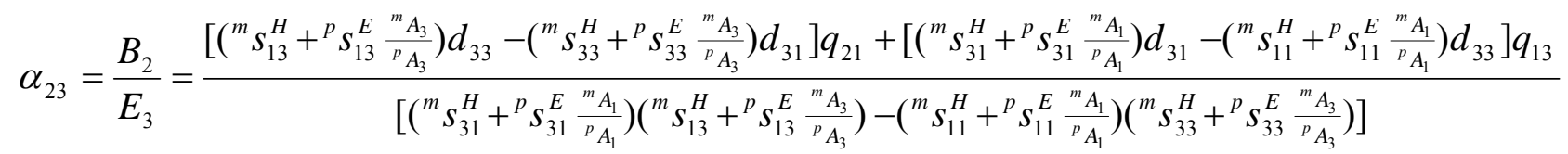

$$
\begin{aligned}
& \alpha_{33}=\frac{B_{3}}{E_{3}}=\frac{\left[\left({ }^{m} S_{13}^{H}+{ }^{p} S_{13}^{E} \frac{{ }^{m} A_{3}}{{ }^{p} A_{3}}\right) d_{33}-\left({ }^{m} s_{33}^{H}+{ }^{p} S_{33}^{E} \frac{{ }^{m} A_{3}}{{ }^{p} A_{3}}\right) d_{31}\right] q_{31}+\left[\left({ }^{m} S_{31}^{H}+{ }^{p} S_{31}^{E} \frac{{ }^{m} A_{1}}{{ }^{p} A_{1}}\right) d_{31}-\left({ }^{m} s_{11}^{H}+{ }^{p} S_{11}^{E} \frac{{ }^{m} A_{1}}{{ }^{p} A_{1}}\right) d_{33}\right] q_{33}}{\left[\left({ }^{m} S_{31}^{H}+{ }^{p} S_{31}^{E} \frac{{ }^{m} A_{1}}{{ }^{p} A_{1}}\right)\left({ }^{m} S_{13}^{H}+{ }^{p} S_{13}^{E} \frac{{ }^{m} A_{3}}{{ }^{p} A_{3}}\right)-\left({ }^{m} s_{11}^{H}+{ }^{p} S_{11}^{E} \frac{{ }^{m} A_{1}}{{ }^{p} A_{1}}\right)\left({ }^{m} S_{33}^{H}+{ }^{p} S_{33}^{E} \frac{{ }^{m} A_{3}}{{ }^{p} A_{3}}\right)\right]}
\end{aligned}
$$

\section{DISCUSSION}

For single phase $\mathrm{ME}$ materials such as $\mathrm{Cr}_{2} \mathrm{O}_{3}$ and $\mathrm{BiFeO}_{3}$, the Gibbs free energy of the material contains a coupled electric magnetic term $-\alpha_{\mathrm{ij}} \mathrm{E}_{\mathrm{i}} \mathrm{H}_{\mathrm{j}}$, so from thermodynamics it can be obtained that the $\mathrm{ME}_{\mathrm{E}}$ coefficient equals the $\mathrm{ME}_{\mathrm{H}}$ coefficients $^{6}$. However, this is not the case for composite materials where ME effect is a product property. For a MS/PE composite, the free energy of the system is the sum of the free energy of the MS and PE materials, where there is no apparent coupling between the electric and magnetic field. Their indirect coupling is through the coupling of the stress and strain at the interface of the two materials. For a loss free system with perfect strain coupling between the MS and PE materials, it is still 
possible that the $\mathrm{ME}_{\mathrm{E}}$ coefficient equals the $\mathrm{ME}_{\mathrm{H}}$ coefficients under certain conditions but this is not as straightforward as for the single phase ME materials.

Defining the magnetoelectric Oersted coefficient as $\alpha_{\mathrm{H}, \mathrm{ij}}=\alpha_{\mathrm{ij}} / \mu_{0} \mu_{\mathrm{r}}=\mathrm{H}_{\mathrm{i}} / \mathrm{E}_{\mathrm{j}}$ where $\mu_{0}$ is the permeability in vacuum and $\mu_{\mathrm{r}}$ is the relative permeability of air, then $\alpha_{\mathrm{H}, \mathrm{ij}}$ has the unit Oe $\mathrm{cm} / \mathrm{V}$ and $\mathrm{H}_{\mathrm{i}}$ is equal to the measured magnetic field immediately outside the MS material along the $\mathrm{i}$ direction. However, if the cross sectional area ${ }^{\mathrm{m}} \mathrm{A}_{3}$ of the sample is smaller than the area of the detector, or the induced magnetization is detected by a pickup coil around the sample, the measured response is proportional to the total induced flux, e.g., $B_{3}{ }^{m} A_{3}$. The dependence of $\alpha_{H, i j}$ as a function of the ratio of the cross sectional areas (or of the thickness ratio if the widths are the same for the PE and MS layers) can be calculated using the above results.

Equations (5), (6) and (7) indicate that, generally speaking, the induced magnetization is three dimensional. However, for MS materials with strong anisotropy, i.e., $\mathrm{q}_{33}>>$ $\mathrm{q}_{11}$, and $\mathrm{q}_{33}>>\mathrm{q}_{22}$, the main magnetization occurs along the direction 3 .

At the conditions $\frac{{ }^{m} A_{2}}{{ }^{p} A_{2}}=\frac{{ }^{m} A_{1}}{{ }^{p} A_{1}}$ for the case (i),

$$
\alpha^{E}{ }_{33}=\frac{2 d_{31} q_{31}^{p} v}{\left({ }^{m} s_{12}^{H}+{ }^{m} s_{11}^{H}+{ }^{p} s_{12}^{E m} v+{ }^{p} s_{11}^{E m} v\right)}
$$

this compares to Harshe's expression for the $\mathrm{ME}_{\mathrm{H}}$ effect

$$
\begin{aligned}
& \alpha^{H}{ }_{33}=\frac{-2 d_{31} q_{31}{ }^{m} v}{\left({ }^{m} s_{11}^{H}+{ }^{m} s_{12}^{H}\right)^{p} v+\left({ }^{p} s_{11}^{E}+{ }^{p} s_{12}^{E}\right)^{m} v-2\left(d_{31}\right)^{2}{ }^{m} v /{ }^{p} \varepsilon_{33}} \\
& =\frac{-2 d_{31} q_{31}{ }^{m} v}{\left({ }^{m} s_{11}^{H}+{ }^{m} s_{12}^{H}\right)^{p} v+\left({ }^{p} s_{11}^{E}+{ }^{p} s_{12}^{E}\right)^{m} v-2\left(k_{31}\right)^{2}{ }^{p} s_{11}^{E}{ }^{m} v}
\end{aligned}
$$


where $\mathrm{k}_{31}$ is the coupling coefficient for the piezoelectric phase. It can be seen that both the $\mathrm{ME}_{\mathrm{E}}$ coefficient and the $\mathrm{ME}_{\mathrm{H}}$ coefficients are proportional to $d_{31} q_{31}$, the product of piezoelectric and piezomagnetic coefficients, but they are not equal to each other at all conditions, as is the case for single phase ME materials.

For numerical calculations, we assume $\frac{{ }^{m} A_{2}}{{ }^{p} A_{2}}=\frac{{ }^{m} A_{1}}{{ }^{p} A_{1}}$ for the case (i) and $\frac{{ }^{m} A_{3}}{{ }^{p} A_{3}}=\frac{{ }^{m} A_{1}}{{ }^{p} A_{1}}$ for (iii), and $\frac{{ }^{m} A_{3}}{{ }^{p} A_{1}}=\frac{{ }^{m} A_{1}}{{ }^{p} A_{2}}$ for case (ii). If the PE material is PZT and the MS material is TFD, for the material parameters given in Table II, Fig. 2a shows the ME coefficient $\alpha_{33}$ as a function of volume fraction ${ }^{\mathrm{m}} \mathrm{v}={ }^{\mathrm{m}} \mathrm{A} /\left({ }^{\mathrm{m}} \mathrm{A}+{ }^{\mathrm{p}} \mathrm{A}\right)$ assuming the widths are the same for the MS and PE layers. The total induced flux per unit electric field $\alpha_{33}{ }^{m} v=B_{3}{ }^{m} v_{3} / E_{3}$ as a function of $m_{v}$ is shown in Fig. $2 b$. It can be seen that the inplane configuration has the highest magnetoelectric response, and the transverse orientation has the lowest among the three cases discussed.

We reported ${ }^{22}$ preliminary measurement results of the magnetoelectric Oersted coefficient $\alpha_{\mathrm{H}, 33}=15.4 \mathrm{Oe} /(28 \mathrm{KV} / \mathrm{m})=55 \mathrm{Oe} \mathrm{cm} / \mathrm{KV}$ and $\mathrm{ME}$ coefficient $=\mathrm{M} / \mathrm{E}=5.7$ $\mathrm{x} 10^{-8}(\mathrm{~s} / \mathrm{m})$ for the tri-layer laminated composites of TFD/PZT/TFD and $\mathrm{PZT} / \mathrm{TFD} / \mathrm{PZT}$ at the fundamental mechanical resonant frequency. The samples were configured as in case (ii). Using the above formula, the calculated $\alpha_{33}=8.3 \times 10^{-8}$ $(\mathrm{s} / \mathrm{m})$, and $\alpha_{\mathrm{H}, 33}=\alpha_{33} / \mu_{0} \mu_{\mathrm{r}}=6.6 \times 10^{-2}(\mathrm{~A} / \mathrm{m} / \mathrm{V} / \mathrm{m})=80 \mathrm{Oe} \mathrm{cm} / \mathrm{KV}$. Both the theoretical and experimental values are more than 4 orders of magnitude greater than the value for the single phase material $\mathrm{Cr}_{2} \mathrm{O}_{3}$. Although the experimental value agrees well with the calculated one, strictly speaking this theory is not applicable at resonant frequency and therefore they are not comparable. The ME response at resonant frequency could 
be a few times larger than the non-resonant frequency response. However this discrepancy between theory and experiment for ME response as a product property is not unusual ${ }^{8,11,23,30}$. The main reason is believed to be the assumption of perfect coupling at the interface in the theory, which is probably not true in practice.

The ME effect as a product property in multilayer PE/MS thin films is a promising research subject ${ }^{15}$. However the giant MS material TFD may not be the most suitable material for this application for reasons such as the applicable production method, material porosity and brittleness. Magnetostrictive oxides such as Ni and Co ferrite are often the chosen MS materials in these situations. We have also estimated the $\mathrm{ME}_{\mathrm{E}}$ coefficient for the $\mathrm{PZT} / \mathrm{CoFe}_{2} \mathrm{O}_{4}$ bilayer composite (material properties are summarised in Table II). This composite can be prepared in the form of thin films by the sol-gel method. Fig. 3a shows the $\mathrm{ME}_{\mathrm{E}}$ coefficient $\alpha_{33}$ as a function of volume fraction ${ }^{\mathrm{m}} \mathrm{v}={ }^{\mathrm{m}} \mathrm{A} /\left({ }^{\mathrm{m}} \mathrm{A}+{ }^{\mathrm{p}} \mathrm{A}\right)$ if the widths are the same for the MS and PE layers. The total induced flux per unit electric field $\alpha_{33}{ }^{m_{v}}=B_{3}{ }^{m_{2}} / E_{3}$ as a function of ${ }^{m} v$ is shown in Fig. 3b. It can be seen that there is little difference between the in-plane and transverse configurations for the ME response, and the longitudinal orientation has the lowest response among the three boundary conditions. Notice also, the magnitude of the ME response is about $1 / 10$ th that of the TFD/PZT composite.

\section{CONCLUSIONS}

Theoretical modelling of the $\mathrm{ME}_{\mathrm{E}}$ magnetoelectric effect for 2-2 connectivity composites has been developed for three different boundary conditions by assuming 
perfect coupling at the interface. The calculated ME coefficients using the material properties of PZT and Terfenol-D are a few orders larger than those of any single phase ME materials.

\section{ACKNOWLEDGEMENTS}

This work was financially supported by the UK EPSRC under Platform Grants

GR/R92448 (Thin film ferroelectrics for nanotechnology applications) and

EP/D506638/1 (Nanoscale Multifunctional Ferroic Materials and Devices) and EP/C519426/1 (Magnetoelectric Materials and Devices). Prof. R.W. Whatmore of Tyndall National Institute, Ireland, Dr P. Record and Mr. C. Popov of Heriot-Watt University and Dr. C. Shaw of Cranfield University are thanked for helpful discussions. 


\section{References}

1. L.D. Landau and E. M. Lifshitz, "Electrodynamics of Continous Media," Pergamon Press, Oxford (1960) (Translation of Russian Edition, 1958).

2. D.N. Astrov, Zh. Exp. Teor. Fiz., 37, 881 (1959) [Soviet Phys.-JETP, 10, 628 (1960)].

3. V.J. Folen, G.T. Rado and E.W. Stalder, Phys. Rew. Lett., 6, 607 (1961).

4. G.J. Rado and V.J. Folen, J. Appl. Phys., 33, 1126 (1962).

5. G.T. Rado, J.M. Ferrari, and W.G. Maisch, Phys. Rew. B29, 4041 (1984).

6. $\quad$ K. Agyei and J. L. Birman, J. Phys.: Condens. Matter., 2, 3007 (1990).

7. J. van Suchtelen, Philips Res. Rep., 27, 28 (1972).

8. J. van den Boomgaard, D.R. Terrell, R.A.J. Born and H.F.J.I. Giller, J. Mater. Sci., 9, 1705 (1974); A.M.J.G. van Run, D.R. Terrell, and J.H. Scholing, J. Mater. Sci., 9, 1710 (1974).

9. C.W. Nan, N. Cai, L. Liu, J. Zhai, Y. Ye and Y. Liu, J. Appl. Phys., 94(9), $5930(2003)$.

10. X. Liu, S. Fu and C. Huang, Mater. Sci. \& Engin., B121, 255 (2005).

11. G. Srinivasan, E.T. Rasmussen, J. Gallegos, R. Srinivasan, Yu.I. Bokhan, and V.M. Laletin, Phys. Rev. B64, 214408 (2001)

12. J. Ryu, A.V. Carazo, K. Uchino, and H.E. Kim, Jpn. J. Appl. Phys., Part 1, 40, $4948(2001)$.

13. U. Laletsin, N. Padubnaya, G. Srinivasan and C.P. Devreugd, Appl. Phys. A78, 33 (2004).

14. H. Chang, MSc thesis, Cranfield University, 2006. 
15. M. Fiebig, J. Phys. D: Appl. Phys., 38, R123 (2005) and the references therein.

16. S. Dong, J. Li, and D. Viehland, App. Phys. Lett. 83, 11 (2003).

17. S. Dong, J. Li, and D. Viehland, App. Phys. Lett. 86, 182506 (2005).

18. N. A. Spaldin and M. Fiebig, Science, 309, 391 (2005).

19. H. Ohno, D. Chiba, F. Matsukura, T. Omiya, E. Abe, T. Diett, Y. Ohno and K. Ohtani, Nature, 408, 944 (2000).

20. T. Lottermoser, T. Lonkal, U. Amann, D. Hohlwein, J. Ihringer and M. Fiebig, Nature, 430, 541 (2004).

21. H. Zheng et al., Science, 303, 661 (2004).

22. P. Record, C. Popov, J. Fletcher, E. Abraham, Z. Huang, H. Chang and R.W. Whatmore, submitted to Sensors and Actuators A-Physical.

23. G. Harshe, J.P. Dougherty, and R.E. Newnham, Int. J. Appl. Electromagn. Mater., 4, 145(1993).

24. M. Bichurin, V. Petrov and G. Srinivasan, J. Appl. Phys., 92, 7681 (2002).

25. M. Bichurin, V. Petrov and G. Srinivasan, Ferroelectrics, 280, 165 (2002).

26. M. Bichurin, V. Petrov, and G. Srinivasan, Phys. Rev. B, 68, 054402 (2003).

27. M. J. Dapino, R.C. Smith, F.T. Calkins and A.B. Flatau, J. Intell. Mater. Sys. \& Struct., 13, 737 (2002)

28. G. Engdahl, "Handbook of Giant Magnetostrictive Materials", Academic Press, 2000. p.175.

29. J. Kim and E. Jung, Smart Mater. Struct. 14 (2005) 1273-1280.

30. S. Dong, J. Li, and D. Viehland, IEEE Trans. Ultras. Ferro. and Freq. Contr., 51, 793 (2004). 
Table I: Some symmetry relations of non-zero coefficients of piezoelectric and piezomagnetic phases.

\begin{tabular}{|l|l|}
\hline \multicolumn{2}{|c|}{ Piezoelectric phase } \\
\hline piezoelectric coefficients & Compliance coefficients \\
\hline $\mathrm{d}_{31}=\mathrm{d}_{32}, \mathrm{~d}_{13}=\mathrm{d}_{23}$ & ${ }^{\mathrm{p}} \mathrm{s}_{11}={ }^{\mathrm{p}} \mathrm{s}_{22}$ \\
\hline $\mathrm{d}_{21}=\mathrm{d}_{12}$ & ${ }^{\mathrm{p}} \mathrm{s}_{12}={ }^{\mathrm{p}} \mathrm{s}_{21}$ \\
\hline $\mathrm{d}_{11}=\mathrm{d}_{22}$ & ${ }^{\mathrm{p}} \mathrm{s}_{13}={ }^{\mathrm{p}} \mathrm{s}_{31}={ }^{\mathrm{p}} \mathrm{s}_{23}={ }^{\mathrm{p}} \mathrm{s}_{32}$ \\
\hline piezomagnetic coefficients & ${ }^{\text {Compliance coefficients }}$ \\
\hline $\mathrm{q}_{31}=\mathrm{q}_{32}, \mathrm{q}_{13}=\mathrm{q}_{23}$ & ${ }^{\mathrm{m}} \mathrm{s}_{11}={ }^{\mathrm{m}} \mathrm{s}_{22}$ \\
\hline $\mathrm{q}_{21}=\mathrm{q}_{12}$ & ${ }^{\mathrm{m}} \mathrm{s}_{12}={ }^{\mathrm{m}} \mathrm{s}_{21}$ \\
\hline $\mathrm{q}_{11}=\mathrm{q}_{22}$ & ${ }^{\mathrm{m}} \mathrm{s}_{13}={ }^{\mathrm{m}} \mathrm{s}_{31}={ }^{\mathrm{m}} \mathrm{s}_{23}={ }^{\mathrm{m}} \mathrm{s}_{32}$ \\
\hline
\end{tabular}


Table II: Material property parameters used for the numerical calculation for lead zirconate titanate (PZT) and Terfenol-D (TFD) and $\mathrm{CoFe}_{2} \mathrm{O}_{4} .\left(\mu_{0}=1.257 \times 10^{-6} \mathrm{Vs} / \mathrm{Am}\right.$, $\mu_{\mathrm{r}}=1$.)

\begin{tabular}{|c|c|c|c|}
\hline & $\mathrm{PZT}^{\mathrm{a}}$ & TFD & ${ }^{\mathrm{d}} \mathrm{CoFe}_{2} \mathrm{O}_{4}$ \\
\hline $\mathrm{s}_{11}\left(10^{-12} \mathrm{~m}^{2} / \mathrm{N}\right)$ & 17 & $25^{b}$ & 6.5 \\
\hline $\mathrm{s}_{12}\left(10^{-12} \mathrm{~m}^{2} / \mathrm{N}\right)$ & -5.8 & $-1.8^{\mathrm{b}}$ & -2.4 \\
\hline $\mathrm{s}_{13}\left(10^{-12} \mathrm{~m}^{2} / \mathrm{N}\right)$ & -8.8 & $-16.7^{b}$ & -2.4 \\
\hline $\mathrm{s}_{33}\left(10^{-12} \mathrm{~m}^{2} / \mathrm{N}\right)$ & 22.9 & $40^{\mathrm{b}}$ & 6.5 \\
\hline $\mathrm{d}_{31}\left(10^{-12} \mathrm{~m} / \mathrm{V}\right)$ & -243 & & \\
\hline $\mathrm{d}_{33}\left(10^{-12} \mathrm{~m} / \mathrm{V}\right)$ & 574 & & \\
\hline $\mathrm{q}_{31}\left(10^{-9} \mathrm{~m} / \mathrm{A}\right)$ & & $-53^{c}$ & 0.56 \\
\hline $\mathrm{q}_{33}\left(10^{-9} \mathrm{~m} / \mathrm{A}\right)$ & & $110^{\mathrm{c}}$ & -1.9 \\
\hline
\end{tabular}

a http://www.ferroperm-piezo.com/, data for Pz29.

${ }^{\mathrm{b}}$ Ref. 29.

${ }^{\mathrm{c}}$ Ref. 28.

${ }^{\mathrm{d}}$ Ref. 26.

Figure Captions 
1. Schematic diagrams of magnetoelectric bilayer composites of piezoelectric (PE) and magnetostrictive (MS) materials with 2-2 connectivity for (I) longtitudinal, (II) transverse and (III) in-plane configurations. The electric polarisation $\boldsymbol{\delta E}$ and magnetic DC bias $\mathbf{H}_{\mathbf{0}}$ and easy magnetisation directions are indicated in the diagrams. Cartesian coordinate systems for the PE and MS layers respectively (or for both) are shown on the right-side for each case.

2. $\mathrm{ME}_{\mathrm{E}}$ coefficient (a) $\alpha_{33}$ and (b) $\alpha_{33}{ }^{\mathrm{m}} \mathrm{v}$ as functions of volume fraction ${ }^{\mathrm{m}} \mathrm{v}={ }^{\mathrm{m}} \mathrm{A} /($ ${ }^{\mathrm{m}} \mathrm{A}+{ }^{\mathrm{p}} \mathrm{A}$ ) for (i) longtitudinal, (ii) transverse and (iii) in-plane configurations when the PE material is PZT and the MS material is Terfenol-D. It can be seen that the in-plane configuration has the highest magnetoelectric response, and the transverse orientation has the lowest among the three cases. Material parameters used for the calculation are given in Table II.

3. $M E_{E}$ coefficients (a) $\alpha_{33}$ and (b) $\alpha_{33}{ }^{m} v$ as functions of volume fraction $\mathrm{m}_{\mathrm{v}}={ }^{\mathrm{m}} \mathrm{A} /\left({ }^{\mathrm{m}} \mathrm{A}+{ }^{\mathrm{p}} \mathrm{A}\right)$ for (i) longtitudinal, (ii) transverse and (iii) in-plane configurations when the PE material is PZT and the MS material is $\mathrm{CoFe}_{2} \mathrm{O}_{4}$. It can be seen that there is little difference between the in-plane and transverse configurations for the ME response, and the longitudinal orientation has the lowest response among the three cases. Material parameters used for the calculation are given in Table II. 


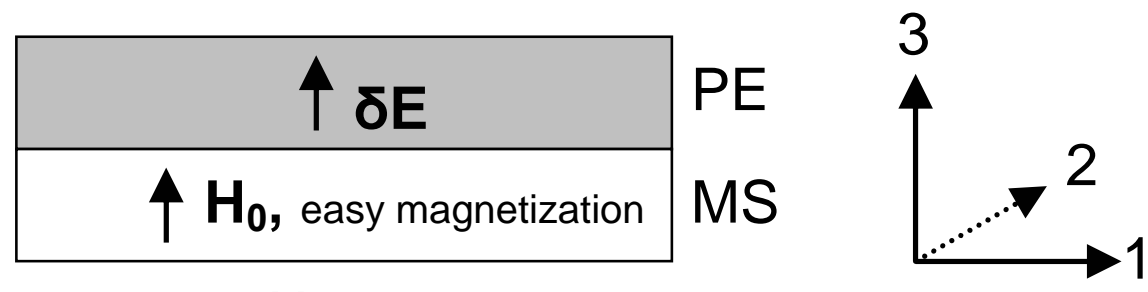

(I)
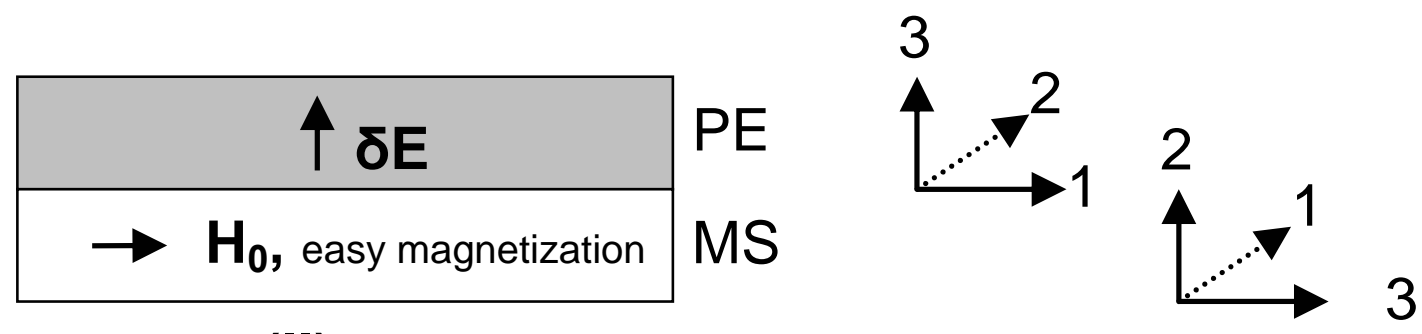

(II)
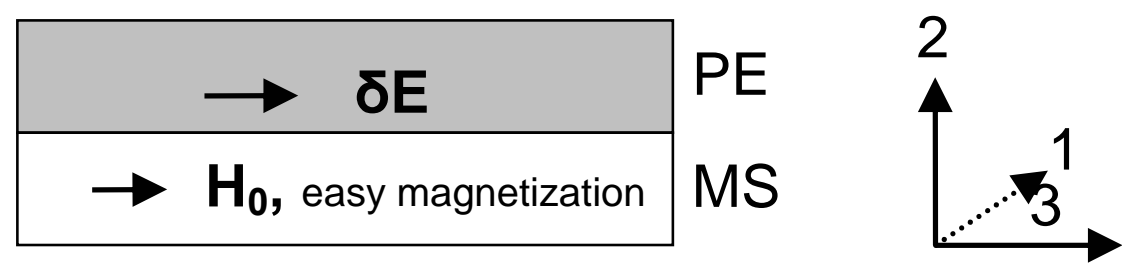

(III)

Fig. 1 

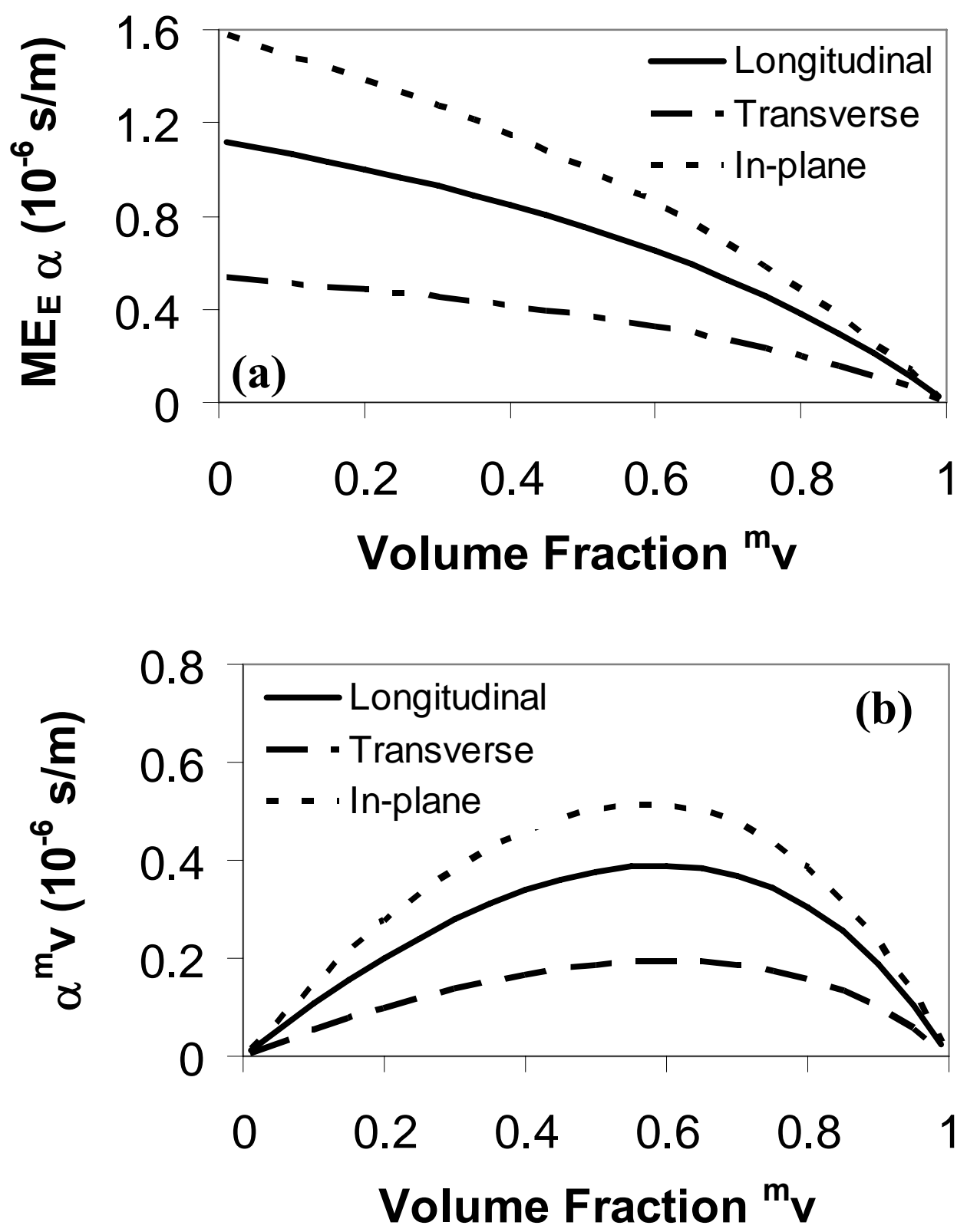

Fig. 2 

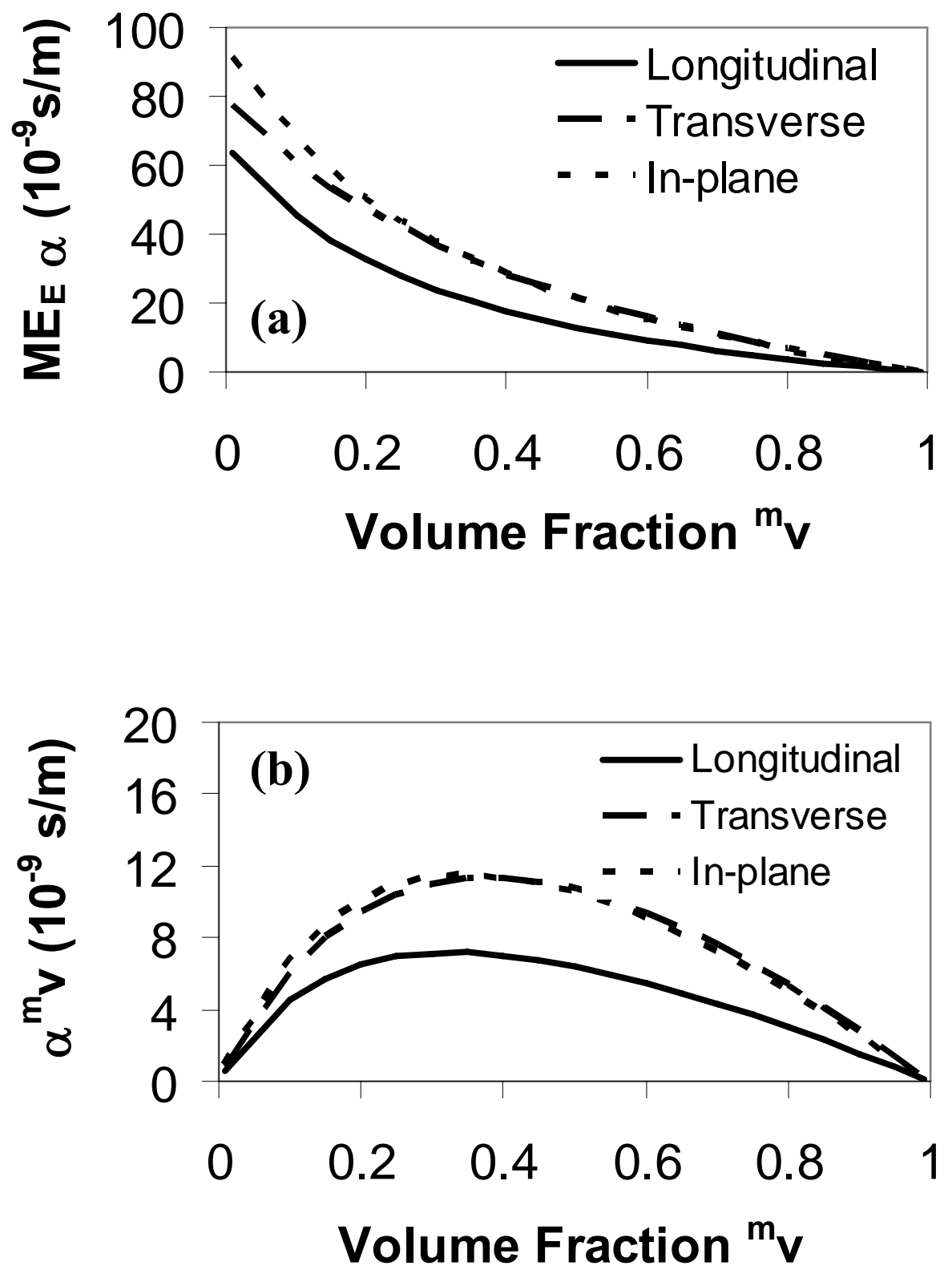

Fig. 3 